\title{
Sexualidad y discapacidad: Síndrome de Mayer-Rokitansky-Küster-Hauser
}

\author{
Fernández Hawrylak, María \\ Departamento de Ciencias de la Educación. Universidad de Burgos \\ mfernandez@ubu.es \\ García Peral, Elisabet \\ Departamento de Ciencias de la Educación. Universidad de Burgos \\ egp0044@alu.ubu.es \\ Armas Junco, Laura \\ Departamento de Ciencias de la Educación. Universidad de Burgos \\ larmas@ubu.es
}

\begin{abstract}
RESUMEN
El síndrome de Mayer-Rokitansky-Küster-Hauser (MRKH), comúnmente denominado síndrome de Rokitansky, es una rara anomalía congénita del tracto genital caracterizada por la ausencia de útero y vagina en mujeres fenotípicamente normales. Se considera probable que responda a una etiología poligénica multifactorial que produce una inadecuada fusión de los conductos de Müller durante el desarrollo embrionario. Se puede distinguir entre síndrome MRKH tipo I, con una aplasia únicamente vaginal y tipo II, asociado a otras malformaciones del sistema renal y esquelético. Esta anomalía anatómica implica dificultades en las relaciones sexuales, infertilidad, carga emocional, cuestiones de identidad, confusión en los roles sociales y sexuales y creencias negativas sobre sí misma. El impacto psicosocial que el diagnóstico del síndrome conlleva, requiere de apoyo psicológico, farmacológico y de sistemas de orientación tanto para la mujer como para su familia debido a las repercusiones en múltiples dimensiones de la vida de las afectadas. Se presenta una investigación utilizando como referente metodológico la historia de vida, con el objetivo de comprender la vivencia de una mujer con MRKH tipo II integrando aquellos elementos del pasado que se valoraron relevantes para describir, entender o representar su situación actual en relación a sus expectativas vitales futuras. Se consideró el método biográfico-narrativo como el más adecuado para llevar a cabo el estudio. Para la redacción de la narrativa se realizaron entrevistas semiestructuradas y se analizaron relatos autobiográficos e informes médicos y escolares. El análisis siguió la ordenación y clasificación de la información por unidades de significado para proceder a su interpretación. Los resultados confirman el impacto en la mujer con una fuerte repercusión en el desarrollo sexual, lo que implica seguir investigando en la inacabada tarea de la educación sexual para el acceso universal a una educación integral de la sexualidad.
\end{abstract}

PALABRAS CLAVE: Mayer-Rokitansky-Küster-Hauser; enfermedades raras; historia de vida; educación sexual, sexualidad.

\begin{abstract}
Mayer-Rokitansky-Küster-Hauser (MRKH) syndrome, also known as Müllerian agenesis, is a rare congenital malformation of the genital tract characterised by a missing uterus and vagina in women with normal female phenotype. It is believed to arise from a multifactorial polygenic aetiology that causes inadequate fusion of the Müllerian duct during embryo development. MRKH is divided into type I, with only vaginal aplasia, and type II, associated with other malformations of the renal and skeletal systems. This
\end{abstract}


anatomic anomaly results in difficulties having sexual relations, infertility, emotional burden, identity issues, confusion regarding social and sexual roles and negative selfimage. The psychosocial impact of being diagnosed with this syndrome requires psychological and pharmacological support, as well as guidance systems for the woman and her family, given the repercussions on many facets of the patient's life. We are presenting a study using the life-history method in order to understand the experience of a woman with type-II MRKH by integrating elements of her past that are deemed relevant to describe, understand or represent her current situation with regard to her expectations for the future. The biographic-narrative method was chosen as the most appropriate for this study. To draft the narrative, we conducted semi-structured interviews and analysed autobiographical stories, medical charts and school reports. The analysis used units of meaning to organise and classify the information for interpretation. The results confirm the impact on women with strong repercussions on sexual development, which implies a need to continue studying the unfinished task of sexual education with universal access to comprehensive education on sexuality.

KEYWORDS: Mayer-Rokitansky-Küster-Hauser; rare diseasses; life history; sexual education; sexuality. 


\section{INTRODUCCIÓN}

El síndrome de Mayer-Rokitansky-Küster-Hauser, también conocido como aplasia o agenesia mülleriana pero más comúnmente denominado síndrome de Rokitansky, es una rara anomalía congénita del tracto genital; una enfermedad congénita caracterizada por la ausencia de útero y vagina en mujeres fenotípicamente normales, con cariotipo $46 \mathrm{XX}$, con un estado endocrino normal (Páez-López et al., 2013). Aparece en 1 de cada 4.5005.000 mujeres y aunque se desconoce su etiología, la creciente comunicación de casos familiares hace pensar que pueda tener una base genética (Arce-Segura et al., 2016; Ramírez-Isarraraz et al., 2020).

El síndrome de MRKH se caracteriza por una aplasia congénita del útero y la parte superior (2/3) de la vagina en mujeres que muestran un desarrollo normal de las características sexuales secundarias (Morcel et al., 2007). Esta malformación de los genitales femeninos con aplasia o hipoplasia del útero y la vagina, es decir, con falta de desarrollo o desarrollo escaso o incompleto de estos órganos reproductores internos de la mujer, se produce por una inadecuada fusión de los conductos de Müller. En cuanto a su etiología, actualmente se asume una patogénesis multifactorial en donde diferentes regiones cromosómicas y estructuras genéticas podrían estar relacionadas con la aparición de esta enfermedad. Clínicamente aparece por la ausencia de la menarquia en la pubertad (Brucker et al., 2020).

Aproximadamente el $25-50 \%$ de las pacientes asocian anomalías del tracto genitourinario (agenesia renal unilateral, riñón pélvico o alteraciones del sistema excretor), el 10-15\% alteraciones esqueléticas en costillas, columna y extremidades, y en el $2-7 \%$ de las mujeres se aprecia un útero (rudimentario u obstruido) con endometrio funcionante (Arce-Segura et al., 2016).

Se puede distinguir entre MRKH tipo I y tipo II. El síndrome MRKH de tipo I se diagnostica con una aplasia únicamente vaginal y uterina mientras que el tipo II se asocia con otras malformaciones, especialmente del sistema renal y esquelético (Brucker et al., 2020). La agenesia unilateral renal (ausencia en el nacimiento de un riñón), la anomalía de Klippel-Feil (fusión congénita de 2 o más vértebras cervicales), escoliosis, defectos auditivos y más raramente, anomalías cardíacas son algunos ejemplos. La aplasia úterovaginal aislada también se denomina secuencia de Rokitansky o síndrome de MRKH de tipo I (aislado). La aplasia incompleta y/o asociada con otras malformaciones, generalmente se conoce como asociación MURCS (o síndrome MRKH tipo II) (Morcel et al., 2007).

En el diagnóstico, uno de los métodos utilizados es el examen por ultrasonido ya que muestra el nivel superior de la vagina y la presencia o ausencia del útero. También permite detectar anomalías coexistentes en los riñones o en la vejiga. En muchos casos, el ultrasonido puede resultar no concluyente o incompleto sobre todo para la distinción de los 2 tipos de síndromes. La resonancia magnética es más sensible que el ultrasonido y permite analizar las alteraciones en los conductos de Müller, que están presentes en el 90\% de las personas que padecen esta enfermedad (Liszewska-Kaplon et al., 2020).

El tratamiento médico del síndrome MRKH supone la creación de una neovagina a través de procedimientos quirúrgicos y no quirúrgicos. Los enfoques no quirúrgicos implican el agrandamiento vaginal con dilatadores, utilizando una presión simple gradual con dispositivos cada vez más grandes. La dispareunia y la obstrucción del tracto urinario son 
algunas complicaciones que pueden surgir tras estos procedimientos. En general, las mujeres prefieren los procedimientos quirúrgicos ya que las intervenciones son rápidas y menos frustrantes que los métodos no quirúrgicos, que pueden ser difíciles de practicar también debido a limitaciones culturales y sociales, y los sentimientos de vergüenza (Dabaghi et al., 2019). Durante la última década, se han producido avances en la investigación, principalmente en los campos de la genética, el manejo no quirúrgico y el trasplante de útero como primer tratamiento de fertilidad disponible (Krogh et al., 2020).

El impacto psicosocial que el diagnóstico del síndrome conlleva para la paciente y para su entorno, requiere de apoyo psicológico, farmacológico y sistemas de orientación, tanto para la afectada como para su familia debido a que este diagnóstico tiene repercusiones en la reproducción futura y en otras dimensiones de la vida de las mujeres afectadas.

Para las mujeres que padecen esta anomalía congénita del tracto genital, en la transición de la niñez a la edad adulta, el diagnóstico supone una gran carga emocional y mental, sobre todo por la infertilidad y la falta de experiencias sexuales, lo que implica cambios y dificultades en el desarrollo sexual femenino y en la identidad (Brucker et al., 2020). Durante la adolescencia, en circunstancias adecuadas compartir el diagnóstico con los compañeros puede ser una fuente de apoyo y fortalecer las relaciones personales. Las preocupaciones iniciales generalmente se centran en la adaptación psicológica de la paciente y su familia (Ernst et al., 2016). También supone confusión en los roles sociales y sexuales y creencias negativas sobre sí misma. Los síntomas depresivos aparecen con frecuencia; la tendencia a la depresión y los pensamientos suicidas como reacción al diagnóstico (Chen et al., 2020). Después del diagnóstico, las mujeres con este síndrome también pueden sentir ansiedad por su feminidad, imagen física e infertilidad (Song et al., 2020).

El síndrome de MRKH provoca un fuerte impacto en el desarrollo sexual de las mujeres afectadas, que se ve obstaculizado tras el diagnóstico. El tipo de actividad sexual, la iniciación de la misma y la frecuencia de orgasmos que acompañan a las actividades sexuales es diferente en las mujeres que no lo padecen. Antes del diagnóstico el desarrollo sexual es normativo en cuanto a la autoerótica y la consciencia de la excitación en contacto con el propio cuerpo. Las diferencias comienzan en etapas posteriores del desarrollo sexual en función de la edad del diagnóstico y cuando se producen relaciones con otros; las malformaciones conllevan un retraso en el inicio de la actividad sexual con otra persona, provocando en la mujer ansiedad, vergüenza, disgusto con el propio cuerpo y rechazo a mantener relaciones sexuales con otras personas, disminuyendo, por tanto, la calidad de vida (Beisert et., al 2015). La falta de experiencias sexuales en estas mujeres implica que su desarrollo sexual al igual que su identidad se vea muy afectada llegando incluso a considerase poco o nada mujeres (Brucker et al., 2020). Para que este hecho se minimice lo antes posible y no afecte considerablemente a la salud mental de las pacientes, se ha de realizar con ellas una correcta educación sexual enfocada al autoconocimiento, la aceptación de sí misma y a su propia satisfacción (Sáinz et al., 2011).

La base de una correcta sexualidad en todas las personas y en especial en las mujeres con síndrome de MRKH, debe partir de la premisa de que la sexualidad es parte de la naturaleza humana, por lo que está presente desde que la persona nace y le acompaña en toda su vida. En relación con la educación sexual de las mujeres con síndrome de MRKH, es importante enseñar a desvincular la sexualidad del mantenimiento de relaciones 
sexuales (Rubio, 2020). Además, el acompañamiento durante el proceso de adaptación y autoconocimiento es fundamental para minimizar las implicaciones psicológicas y evitar las malas prácticas relacionadas con la sexualidad.

La etapa de la adolescencia tiene una importancia vital, porque es cuando se produce la estructuración de la personalidad del individuo. En la adolescencia, la limitación de actividades sociales puede afectar a la construcción de la identidad, que se forma con una carga importante de incertidumbre hacia el futuro y de dependencia del entorno (Fernández-Hawrylak, y Grau, 2014). Los intensos cambios que viven y la búsqueda de la identidad contribuyen a que muchas veces se vean perdidos. Por ello, dada la edad en la que se diagnostica este síndrome, generalmente en la adolescencia, es también conveniente que los centros educativos intervengan para apoyar el proceso educativo desde los equipos docentes y de orientación.

El proceso de construcción de la identidad personal se define en función de la relación de la persona consigo misma y con su entorno. Se trata de un proceso dinámico porque los elementos configuradores de la identidad se modifican a lo largo de la vida. Por tanto, la identidad personal no es la suma de recuerdos personales, sino una construcción narrada sobre las vicisitudes de la intención humana organizada en el tiempo (McAdams, 2003). Las personas desarrollan su identidad y el conocimiento de su entorno a partir de la experiencia con su propio cuerpo, emociones, motivaciones, expectativas y de la elaboración de estas experiencias para construir un autoconcepto o imagen de sí mismo. En este proceso la relación con su familia, la escuela y la sociedad la modulan al otorgar experiencias que influyen en desarrollo del concepto de identidad personal (Bajardi, 2015). Esta identidad personal que se va desarrollando durante toda la vida permitirá abordar las experiencias vitales con respecto a la propia persona y a su entorno. Pero ¿cómo una adolescente a la que se ha diagnosticado síndrome MRKH se adapta y reconstruye su identidad? En este trabajo se presenta una investigación utilizando como referente metodológico la historia de vida, con el objetivo de comprender la vivencia de una mujer afectada por síndrome de Rokitansky integrando aquellos elementos del pasado que considera relevantes para describir, entender o representar su situación actual en relación a sus expectativas vitales futuras.

\section{DISEÑO DE LA INVESTIGACIÓN}

\section{METODOLOGÍA}

Se presenta una investigación a partir de una historia de vida que describe las transiciones de unas etapas a otras de una mujer que ha experimentado debido a una enfermedad una trayectoria de vulnerabilidad, exclusión e inclusión en el marco social y educativo, desde su adolescencia hasta su adultez. Dentro de la metodología cualitativa, el enfoque biográfico-narrativo pretende la exploración de los significados profundos de las historias de vida (Bolívar et al., 2001). Además, en la actualidad la investigación biográficonarrativa posee reconocimiento y prestigio suficientes como para poder afirmar que está consolidada y que ha adquirido identidad propia dentro de la investigación cualitativa (Bolívar, y Domingo, 2018). Precisamente por ello se opta por utilizar el método biográfico-narrativo para su aproximación al campo de estudio puesto que la investigación biográfico-narrativa permite que emerjan y se desarrollen perfiles que vinculan estrategias cualitativas de investigación a los actores reales de la vida cotidiana (Bolívar, y Domingo, 2006). En este caso, acercarse a lo que es vivir con Síndrome de Rokitansky a partir de la experiencia y el testimonio de quien lo padece. 
La investigación biográfica no implica la construcción de una historia o biografía con fines terapéuticos o históricos, consiste en reelaborar con nuevas vivencias fragmentos de vida con valor único, pero extrapolables a la comprensión de la realidad común (Cortés, 2011). Los datos provienen, por tanto, de la vida cotidiana, de las explicaciones y reconstrucciones que el sujeto realiza para vivir y sobrevivir diariamente (RuizOlabuénaga, 2012). En las biografías y las historias de vida, el investigador debe obtener datos completos y profundos sobre cómo perciben los individuos los acontecimientos de sus vidas y a sí mismos (Hernández et al., 2014). La historia de vida requiere, por tanto, de la verificación y concordancia de los hechos narrados, a través de otros datos aportados por otros métodos de registro y con material proporcionado por otras personas que conocen al individuo y los lugares y acontecimientos descritos (Martín, 1995).

Siguiendo a Bolívar, y Domingo (2018) se ha procurado tomar en consideración:

1. La determinación del objeto de estudio: al respecto, las investigadoras no hemos encontrado estudios desde el enfoque cualitativo. Además, el tema está teniendo un fuerte impacto en la actualidad debido a los avances médicos.

2. La determinación de la perspectiva analítica: nuestra intención ha sido en todo momento la compresión del problema de investigación a través de una metodología cualitativa a fin de complementar las perspectivas médicas y psicológicas desde las cuales se ha abordado la enfermedad que estudiamos.

3. La determinación de la mirada con la que se aborda el estudio: el trabajo se ha abordado desde una visión de consenso negociada entre la protagonista que habla "de motus propio", como afectada y como investigadora, sus compañeras coinvestigadoras y otros actores de su entorno.

4. La determinación del provecho del relato: dirigido a las asociaciones afines a la enfermedad y a través de la construcción de contenidos relacionados con la educación afectivo-sexual y la discapacidad.

\section{RECOGIDA DE DATOS}

Para la redacción de la narrativa se utilizaron varios instrumentos de recogida de datos y se acudió a diversas fuentes de información. Las entrevistas semiestructuradas fueron las fuentes de información principales para narrar la historia de vida. Pérez (1994) propone delimitar el contenido básico de la historia de vida identificando tres dimensiones: a) las dimensiones básicas de la vida del sujeto, como la biológica, cultural y social; b) los puntos de inflexión o eventos cruciales en los que la persona altera drásticamente sus roles habituales, ya que se enfrenta con una nueva situación o cambia de contexto social; y c) el proceso de adaptación y desarrollo de los cambios, lentos o rápidos, que se van sucediendo en el proceso de su vida. Se realizaron las entrevistas planteando preguntas abiertas desde los ocho apartados o dimensiones contempladas por Atkinson (1998) que aglutinan cuestiones en torno a los cuales se puede vertebrar las entrevistas para realizar una historia de vida: el nacimiento y familia de origen, el escenario cultural y tradicional, los factores sociales, la educación, el amor y el trabajo, los eventos y períodos históricos, la vida interior y espiritualidad y la visión de futuro. Se abordaron temas asociados a la caracterización del propio entrevistado acerca de sí mismo y de experiencias significativas de su vida y entorno. Se llevaron a cabo dos entrevistas a la protagonista, una a su hermana y otra a su madre con distinta con distinta duración (entre media hora y hora y media) en sus domicilios. En todas se pidió a los relatores que nos ofrecieran tanta información como les fuera posible para poder comprender cómo los diferentes aspectos de las tres dimensiones se han visto impactadas por la enfermedad, aunque en otros momentos las intervenciones consistieron en un pedido de interpretación o consideración 
por parte del entrevistado. Se ofreció a los participantes la devolución transcrita de las entrevistas para su lectura. También se analizó un relato autobiográfico previamente publicado en 2018 por la periodista Isabel Gemio, un diario personal, y otras fuentes documentales como informes médicos y escolares.

\section{PROCESAMIENTO Y ORGANIZACIÓN DE LA INFORMACIÓN}

El proceso de elaboración de una historia de vida debe ser algo meditado y bien asentado sobre las convicciones del propio sujeto. En caso de ser elaborado de manera delegada por otra persona o con la colaboración de otras, ha de apoyarse sobre un conocimiento profundo de la historia de vida de esa persona y de su proceso vital. Se obtuvo el permiso de la protagonista y de los participantes a través del consentimiento informado para la participación en el estudio, la grabación y trascripción de las entrevistas, el uso de documentación personal y la publicación de los resultados. Se realizó un análisis categorial-temático que siguió los siguientes pasos: 1) trascripción de las entrevistas; 2) lectura del material; 3 ) primer acercamiento con fines exploratorios (no-estructurado); 4) segmentación del texto de las entrevistas en unidades temáticas; 5) identificación de las categorías presentes dentro de las tres dimensiones y análisis de la historia con integración del material documental. La ordenación y clasificación de la información por unidades de significado, junto con el contraste con los datos procedentes de otras fuentes, permitió triangular la información garantizando el rigor metodológico (Gibbs, 2012). Se han tenido en cuenta los criterios de rigor (Lincoln, y Guba, 1985; Guba, y Lincoln, 1989) para alcanzar la confiabilidad (trustworthiness). Se ha contextualizado la investigación, se han descrito los pasos llevados a cabo para la recogida de datos aportando ejemplos específicos, pudiendo aportarse tanto las transcripciones textuales de las respuestas de los participantes como el resto de los documentos; se ha detallado la metodología, el lugar del estudio, los participantes y los procedimientos utilizados de recolección, análisis e interpretación de datos. Las entrevistas se basaron en la literatura y dos de las investigadoras codificaron de forma independiente las transcripciones reuniéndose posteriormente para discutir los hallazgos y los acuerdos se alcanzaron por consenso entre las tres investigadoras. Los aspectos éticos fueron resguardados de acuerdo a los principios de la Declaración Universal sobre Bioética y Derechos Humanos (UNESCO, 2005).

\section{RESULTADOS}

Seguidamente se presenta un breve balance de los principales resultados obtenidos atendiendo a la categorización procedente de las tres dimensiones que originalmente se acordaron.

\section{- DIMENSIÓN BÁSICA}

La protagonista de esta historia es una mujer nacida en 1984 en una de las provincias de Castilla y León cuya vida transcurre dentro de unos parámetros esperados de cualquier menor de la época. Se describe en términos muy positivos dentro de una familia funcional con un fuerte arraigo hacia las tradiciones y consciente de ser receptora de sólidos valores.

Aunque el contenido explícito del discurso pone de manifiesto recuerdos sobre su infancia vinculados a problemas de salud, más bien propios de la infancia, su actitud abierta tuvo un efecto positivo en sus relaciones sociales caracterizadas por muchas situaciones de participación, sentido de pertenencia y continuidad. Un análisis de las declaraciones revela una trayectoria académica y social sin referencias destacadas durante la infancia. 


\section{- DIMENSIÓN DE CAMBIO}

El verdadero punto de inflexión en la valoración de la salud y de su vida lo constituye el diagnóstico del síndrome de Mayer-Rokitansky-Küster-Hauser:

"Una vez que me diagnosticaron, yo perdí contacto con todos los amigos, no quería tener nada que ver con la gente que conocía de antes del diagnóstico [...]". "Puf, de ser una niña extrovertida, con buenas notas, buena estudiante, me volví introvertida, no quería salir de la cama, cuando salía de la cama era para irme de fiesta y no aparecer por casa en 3 días. Me volví agresiva, me enfadaba por todo. A nada que me decían saltaba y saltaba bien, no de déjame en paz, no, me enfadaba, gritaba, chillaba, rompía cosas". (Ent. 1, protagonista).

Al expresarse sobre sus recuerdos tras el diagnóstico, en relación al proceso de construcción de la identidad en interacción con otros y al sentido de pertenencia a distintos grupos socioculturales con los que compartía características en común, la protagonista habló sobre estrategias vinculadas a la negación y la huida, destacó conductas de agresividad y de riesgo, conflictos familiares, acciones contrapuestas a las cualidades de la protagonista.

Las relaciones de pareja fueron descritas como "tóxicas" junto al mantenimiento de relaciones esporádicas con conocidos en vez de amigos y la evitación de relaciones afectivas íntimas. Argumenta el rechazo de las relaciones íntimas unido a la necesidad de explicar y justificarse para sentirse incluida junto al cuestionamiento de su feminidad y roles sexuales, por ser contrarios a los cánones sociales:

"El no decir lo que yo tengo, el no decir lo que a mí me pasa. Antes de operarme, por ejemplo, el estar con un chico y querer llegar a más y decir: ‘ ¿por qué no quieres?', 'no, no quiero', el no decir, claro, como tú no lo tienes asumido, si tú lo cuentas es ir asumiendo lo que tú tienes. Si no lo cuentas eso no existe, como lo que no ves no existe". [...]. "Porque es una cosa mía, a nadie le interesa o le interesaba, ¿no?, claro en aquel entonces: es una cosa mía, es una cosa que me pasa a mí, soy la única, soy la rara. Si yo lo cuento a lo mejor no me aceptan y yo lo que quiero es sentirme aceptada. Bastante tienes ya con sentirte medio mujer, que se te cae el mundo a los pies cuando te dicen que no puedes tener hijos. En la sociedad siempre lo has visto, ¿qué tienen que hacer las mujeres?, las mujeres tienen hijos, claro, ya no puedes tener hijos, ya no eres una mujer completa. No puedes mantener relaciones sexuales, claro, tú por aquel entonces la sexualidad siempre se asociaba al acto sexual, a mantener relaciones sexuales, al coito, punto y pelota. Ahora te das cuenta que la sexualidad pueden ser las caricias, los besos, el estar abrazados en un sofá tranquilamente pero claro, en aquel entonces con 17 años, 18, quieres experimentar, quieres y no puedes porque no tienes las herramientas, a ver, tienes las herramientas: los besos, las caricias, pero claro, no tienes la otra parte fundamental por así decirlo que es la vagina para poder mantener relaciones sexuales". (Ent. 1, protagonista).

A pesar de gozar de una gran fortaleza y espíritu de superación, su narrativa se vincula a un intenso deseo "de retroceder en el tiempo y volver a ser la misma de antes", de "sentirse sola y necesitar ayuda", de "amar y ser amada", valorando opciones para salir adelante a la vez que se iba deprimiendo (Del Diario personal, 2005). 
La relación con su cuerpo se vio cuestionada y consecuentemente su desarrollo sexual, reflejando tensiones en la revelación de la enfermedad a los otros, pero también una dura adaptación a las limitaciones que la malformación le provocó, que derivaron en múltiples consultas y tratamientos psiquiátricos por distinta sintomatología (Chen et. al, 2000), especialmente por ideas negativas sobre su persona, carencia de voluntad e incapacidad para emocionarse (De Informes de Psiquiatría de la protagonista, 2000). No es hasta 2018 cuando se la valora y en el dictamen se le reconoce un grado de discapacidad del $36 \%$ (Resolución Discapacidad de la protagonista, octubre 2018). Probablemente las dificultades adaptativas se vieron acentuadas por las características propias de una enfermedad rara, además concretada en el tipo II del síndrome, que en su caso se cursa con múltiples hernias y la falta de un riñón (De los Informes Ginecológicos de la protagonista, 2015 y ss.).

Como han demostrado algunos autores (Beisert et., al 2015; Brucker et al., 2020), las consecuencias biológicas de este diagnóstico junto con otras experiencias relacionadas con la sexualidad, apuntan la ausencia de educación sexual y transmite un discurso que enfatiza la necesidad de orientación y apoyo durante su etapa escolar. En relación al periodo escolar, relató anécdotas de tensión y fracasos del sistema educativo en materia de educación sexual:

"La ESO yo la empecé en un instituto público y luego me cambié al internado, entonces, durante el instituto público yo siempre he tenido mucho pecho hasta que me operé en 2007, me hice una reducción de pecho. Estábamos en Educación Física y me agarraron entre 3 y me levantaron la camiseta para verme el pecho, porque claro, con 12 años a lo mejor yo tenía una 95 cuando las demás de mi clase estaban planas, entonces pues como que era la novedad, ese es el peor recuerdo que yo tengo. [...] pero vamos, el peor es que te agarren entre 3 y te levanten la camiseta, se lo digas al profesor y el profesor te diga que no quiere chivatos en clase". (Ent. 1, protagonista).

El tratamiento médico quirúrgico o no quirúrgico debe vincularse también al conocimiento de la sexualidad integral, lo que implica que los profesionales adquieran tanto estas competencias como otras habilidades en relación al trato a los pacientes. Caracterizan los relatos la búsqueda de apoyo, psicológico y real y el reclamo de amabilidad y de comprensión:

"Cuando a mí me operaron hace 20 años me mandaron consoladores, 3 consoladores distintos, ¿vale? Yo, que jamás en la vida había estado en un sexshop, tuve que ir a un sexshop que tenía convenio con la Seguridad Social a comprarlos y me dieron 3: uno más pequeño, uno mediano y uno grande. En el momento en el que el pequeño no te costara metértelo, te tenías que meter el mediano y cuando el mediano dejaba de molestarte, tenías que meterte el grande. Siempre con un gel lubricante. Una vez que el grande, no sé si eran 18 centímetros y no te decían, a ti te decían que te lo tenías que poner y punto. No te decían ni cómo tenías que hacerlo ni por ejemplo estimúlate para que tú estés más a gusto y te cueste menos... no, te lo tenías que poner cuando te ibas a la cama y quitártelo cuando te levantabas". (Ent. 1, protagonista).

"Sí, y los modos de cómo te dicen las cosas los médicos claro. [...]. Tienes esta opción: o te operas o te dilatas'. Luego vas y te miden el éxito de la operación que te hacen que consiste en las veces que te has acostado con alguien o en los centímetros. [...]. 
No se preocupan en cómo estás anímicamente o psicológicamente sino en si lo que ellos te han hecho ha funcionado o no ha funcionado". (Ent. 2, protagonista).

"Lo pasamos muy mal. Ella y los demás. Yo recuerdo que, mi madre y yo estábamos fuera y vimos pasar a la gente. Cerraron la puerta y la oímos llorar y con mi madre la sacamos diciendo que no la tocaba nadie. [...] Los médicos deberían de tener un poco más de delicadeza. No hacerla sentir como que es una persona rara. Tiene una enfermedad rara. Aunque no conozcas la enfermedad tienes que tener sensibilidad cuando le vas a decir las cosas. Son niñas que lo tienen todo el día en su cabeza y tú encima no eres lo más amable”. (Ent. hermana).

\section{- DIMENSIÓN DE ADAPTACIÓN}

Las crisis afectan a la identidad, pero el ser humano interpreta y reelabora los hechos o los acontecimientos. Las creencias negativas de la protagonista sobre sí misma se fueron modificando en un proceso de reencuadre. Refiriéndose a su vida actual, ofrece un relato de resiliencia tras la integración de la enfermedad gracias a la influencia positiva del apoyo familiar y tratando de retomar sus expectativas iniciales:

"De adolescente al principio de la enfermedad era una mierda, no valía para nada. Te vas valorando, te vas dando cuenta que la enfermedad no te define". (Ent. 2, protagonista).

"Pues al principio lo ves mal pero ahora yo veo que la persona que soy yo y lo que he llegado a ser ha sido gracias al síndrome, al darte cuenta de que no eres una mujer a medias, por así decirlo y entonces a ser válida por lo que yo soy, por lo que yo he llegado a ser, por lo que yo hago, no por el hecho de ser Rokitansky". (Ent. 1, protagonista).

"Una vez que ella ya empezó a mejorar pues nosotros ya lo hemos vivido de otra manera" (Ent. madre).

\section{CONCLUSIONES}

Este trabajo pone de manifiesto cómo la identidad es un tema fundamental en diferentes campos, entre ellos el educativo. Su influencia en la construcción de la identidad es importante. En este texto se analiza la historia de vida de una mujer con síndrome de Rokitansky, de "una mujer Roki", evidenciando múltiples aspectos vitales que han influido en la construcción o reconstrucción de su identidad. Estos aspectos están en la base del establecimiento de un punto de vista sobre la construcción de las trayectorias de las identidades de las mujeres con MRKH como un proceso de reencuentro (de autoconocimiento y autoaceptación), pero también de formación y reaprendizajes que hace exigencias a los participantes y a sus entornos de vida. Se ha argumentado cómo estos requisitos son diferentes en función de los ámbitos: el de la propia mujer y su familia, y el educativo, el sanitario y el social. Este proceso tiene por tanto su importancia para la formación de profesionales, destinados también, a convertirse en agentes reflexivos de su propia identidad y de la de su futuro alumnado, pacientes y ciudadanos.

Aunque hemos tratado de profundizar e implicarnos verdaderamente, nos ha resultado difícil equilibrar y relacionar los conocimientos científicos con la experiencia vivida tan amplia alrededor del síndrome de MRKH. Mirado el análisis con retrospectiva, surgen nuevos interrogantes y desafíos como incorporar otras voces, las de otras mujeres 
afectadas y, sobre todo, la de los sanitarios ausentes en esta narrativa al tratarse de una situación que suele replicarse con cierta frecuencia en este ámbito sanitario. La biograficidad del proceso identitario de la protagonista, basado en las dimensiones estudiadas, confirma el impacto del diagnóstico en la mujer y en su entorno con una fuerte repercusión en el desarrollo sexual, lo que implica seguir investigando en la inacabada tarea de la educación sexual para el acceso universal a una educación integral de la sexualidad.

\section{REFERENCIAS BIBLIOGRÁFICAS}

Atkinson, R. (1998). The life story interview. SAGE.

Arce-Segura, L.J., Rodríguez-de Mingoa, E., Díaz-Verab, E., García-Sáncheza, V., y Calle-Romeroa, Y. (2016). Síndrome de Mayer-Rokitansky-Küster-Hauser: a propósito de un caso. Medicina de Familia Semergen, 42(5), e50-e52.

Bajardi, A. (2015). La identidad personal en relación con la educación: características y formación del concepto. REIDOCREA, Monográfico Identidad y Educación, 15, 106114.https://digibug.ugr.es/bitstream/handle/10481/37124/Bajardi_IdentidadPersonal. pdf

Beisert, M.J., Szymańska-Pytlińska, M.E., Kapczuk, K., Chodecka, A., Walczyk-Matyja, K., y Kędzia, W. (2015). Sexual activity of women with Mayer-Rokitansky-KüsterHauser syndrome (MRKHS)-preliminary study. Ginekologia Polska, 86(9), 648-652. https://doi.org/10.17772/gp/57853

Brucker, S.Y., Pösch, L.S., Graf, J., Sokolov, A.N., Schaeffeler, N., Kronenthaler, A., Hiltner, H., Wagner, A., Ueding, E., Rieger, M.A., Schöller, D., Stefanescu, D., Rall, K.K., Wallwiener, D., y Simoes, E. (2020). Rare genital malformations in women's health research: sociodemographic, regional, and disease-related characteristics of patients with Mayer-Rokitansky-Küster-Hauser syndrome. BMC Women's Health, 20(1), 1-10. https://doi.org/10.1186/s12905-020-00969-9

Bolívar, A., y Domingo, J. (2018). La investigación (auto)biográfica y narrativa en España: principales ámbitos de desarrollo en educación. Revista Brasileira de Pesquisa (Auto)Biográfica, 3(9), 796-813. http://doi.org/10.31892/rbpab2525426X.v3.n9

Bolívar, A., y Domingo, J. (2006). La investigación biográfica y narrativa en Iberoamérica: Campos de desarrollo y estado actual. FQS-Forum Qualitative Social Sozialforschung/ Forum: Qualitative Social Research, 7(4). http://www.qualitativeresearch.net/index.php/fqs/article/view/161/357

Bolívar, A., Domingo, J., y Fernández, M. (2001). La investigación biográfico-narrativa en educación. Guía para indagar en el campo. La Muralla.

Cortés, P. (2011). El sentido de las historias de vida en investigaciones socio-educativas. Una revisión crítica. En F. Hernández, J.Ma Sancho, y J.I. Rivas (Coords.), Historias de vida en educación: biografías en contexto (pp. 68-74). ESBRINA-RECERCA.

Chen, N., Song, S., Duan, Y., Kang, J., Deng, S., Pan, H., y Zhu, L. (2020). Study on depressive symptoms in patients with Mayer-Rokitansky-Küster-Hauser syndrome: an analysis of 141 cases. Orphanet Journal of Rare Diseases, 15(1), 1-9. https://doi.org/10.1186/s13023-020-01405-9

Dabaghi, S., Zandi, M., y Ilkhani, M. (2019). Sexual satisfaction in patients with MayerRokitansky-Küster-Hauser syndrome after surgical and non-surgical techniques: A systematic review. International Urogynecology Journal, 30, 353-362. https://doi.org/10.1007/s00192-018-3854-5

Ernst, M.E., Sandberg, D.E., Keegan, C., Quint, E.H., Lossie, A.C., y Yashar, B.M. (2016). The Lived Experience of MRKH: Sharing Health Information with Peers. 
Journal of Pediatric and Adolescent Gynecology, 29(2), 154-158. https://doi.org/10.1016/j.jpag.2015.09.009

Fernández-Hawrylak, M., y Grau, C. (2014). Necesidades educativas, asistenciales y sociales especiales de los niños con enfermedades minoritarias: propuestas para una atención interdisciplinar. Revista de Educación Inclusiva, 7(3), 97-124. https://revistaeducacioninclusiva.es/index.php/REI/article/view/134/128

Gemio, I. (2018). Mujeres en el campo de batalla: Mujeres excepcionales ( $1^{\mathrm{a}}$ ed.). En I. Gemio (Ed.), Mi hijo, mi maestro: Una historia de amor y dolor jamás contada (pp. 263-314). La Esfera de los Libros.

Gibbs, G.R. (2012). El análisis de datos cualitativos en investigación cualitativa. Morata.

Guba, E.G., y Lincoln, Y.S. (1989). Fourth generation evaluation. SAGE.

Hernández, R., Fernández, C., y Baptista, P. (2014). Metodología de la investigación (6 ${ }^{\mathrm{a}}$ ed.). McGraw-Hill.

Krogh, M., Bjørn, M., y Brännström, M. (2020). Mayer-Rokitansky-Küster-Hauser (MRKH) syndrome: a comprehensive update. Orphanet Journal of Rare Diseases, 15(1), 1-16. https://doi.org/10.1186/s13023-020-01491-9

Lincoln, Y.S., y Guba, E.G. (1985). Naturalistic Inquiry. SAGE.

Liszewska-Kaplon, M., Strózik, M., Strózik, M., Kotarski, L., Baglaj, M., y Hirnle, L. (2020). Mayer-Rokitansky-Küster-Hauser syndrome as an interdisciplinary problem. Advances in Clinical and Experimental Medicine, 29(4), 505-511. https://doi.org/10.17219/acem/118850

Martín, A.V. (1995). Fundamentación Teórica y uso de las Historias y Relatos de Vida como técnicas de investigación en Pedagogía Social. Aula, 7, 41-60. https://dialnet.unirioja.es/servlet/articulo?codigo=122506

McAdams, D.P. (2003). Identity and the Life Story. En R. Fivush, y C.A. Haden (Eds.), Autobiographical Memoryand the Construction of a Narrative Self. Developmental and Cultural Perspectives (pp. 187-207). Lawrence Erlbaum.

Morcel, K., Camborieux, L., y Guerrier, D. (2007). Mayer-Rokitansky-Küster-Hauser (MRKH) syndrome. Orphanet Journal of Rare Diseases, 2(13), 1-9. https://doi.org/10.1186/1750-1172-2-13

Páez-López, G., De los Ríos-Posada, J.F., Arango-Martínez, A.M., Castañeda-Roldán, J.D., Serna-Agudelo, E., Vásquez-Ruiz, R., Almanza-Payares, L.A., Calle-Gómez, G.A. (2013). Manejo laparoscópico de miomatosis uterina en paciente con síndrome de Rokitansky. Reporte de un caso y revisión de la literatura. Revista Colombiana de $\begin{array}{lllll}\text { Obstetricia } & y & \text { Ginecología, 64(4), } & \text { 469-474. }\end{array}$ http://www.scielo.org.co/pdf/rcog/v64n4/v64n4a07.pdf

Pérez, G. (1994). Investigación Cualitativa. Retos e interrogantes. Técnicas y análisis de datos (Tomo II). La Muralla.

Ramírez-Isarraraz, C., Rodríguez-Colorado, E.S., Viridiana Gorbea-Chávez, V., Verónica Granados-Martínez, V., Olvera-Delgado, J.J., y Bravo-Canales, G.R. (2020). Síndrome Mayer-Rokitansky-Küster-Hauser con prolapso de órganos pélvicos: reporte de un caso. Ginecología y Obstetricia de México, 88(1), 54-58. https://doi.org/10.24245/gom.v88i1.3485

Rubio, N. (2020). Afectividad y sexualidad en personas con discapacidad. Claves y pistas para familias. Editorial Sexualidad y Discapacidad.

Ruiz-Olabuénaga, J.I. (2012). Metodología de la Investigación Cualitativa. Univ. Deusto.

Sáinz, M., de la Cruz, C., y Rubio, N. (2011). En el plural de las sexualidades. Atender, educar y prestar apoyo a la sexualidad de las personas con discapacidad intelectual. Fundadeps, Real Patronato Sobre Discapacidad. 
Song, S., Chen, N., Duan, Y.P., Kang, J., Deng, S., Pan, H. X., y Zhu, L. (2020). Anxiety symptoms in patients with Mayer-Rokitansky-Küster-Hauser syndrome: a crosssectional study. Chinese Medical Journal, 133(4), 388-394. https://doi.org/10.1097/CM9.0000000000000648

UNESCO - Organización de las Naciones Unidas para la Educación, la Ciencia y la Cultura (2005, octubre). Declaración Universal sobre Bioética y Derechos Humanos. Actas de la $33^{\mathrm{a}}$ Conferencia General. París (Francia). https://unesdoc.unesco.org/ark:/48223/pf0000142825_spa.page=85 